\title{
Summarising Censored Survival Data Using The Mean Residual Life Function
}

\author{
John Hinde, Alberto Alvarez-Iglesias, John Newell and Carl Scarrott
}

The mean residual life (MRL) function provides a clear and simple summary of the effect of a treatment or a risk factor in units of time, avoiding hazard ratios or probability scales that require careful interpretation. Estimation of the MRL is complicated by the upper tail of the survival distribution not being observed as, for example, patients may still be alive at the end of the follow up period. Various approaches have been developed to estimate the mean residual life in the presence of such right censoring. In this work, a novel semiparametric method that combines existing non-parametric methods and an extreme value tail model is presented, where the limited sample information in the tail (prior to study termination) is used to estimate the upper tail behavior. This approach will be demonstrated with simulated and real-life examples.

\footnotetext{
John Hinde

National University of Ireland Galway, Galway, Ireland

e-mail: john.hinde@nuigalway.ie
} 